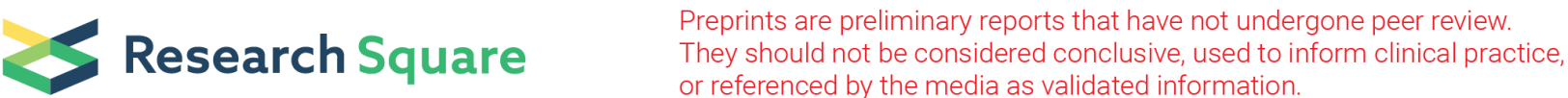

\section{Methylation Analysis in Uygur and Kazak Patients with Hypertension Complicated with Left Ventricular Hypertrophy: Ethnic Difference Based On High- density Genomic DNA Methylation Chips}

\section{Xi Chen}

Xinjiang Medical University Affiliated First Hospital

Na Zhang

Xinjiang Medical University Affiliated First Hospital

Xinjuan Xu ( $\nabla$ zcxu2002@163.com)

Xinjiang Medical University Affiliated First Hospital

Xiaohui Liang

Xinjiang Medical University Affiliated First Hospital

Guangmei Hu

Xinjiang Medical University Affiliated First Hospital

\section{Research article}

Keywords: DNA methylation, hypertension, left ventricular hypertrophy, Uygur and Kazak

Posted Date: July 18th, 2019

DOl: https://doi.org/10.21203/rs.2.11575/v1

License: (c) (i) This work is licensed under a Creative Commons Attribution 4.0 International License.

Read Full License 


\section{Abstract}

Background:This study aimed to analyze the methylation level in Uygur and Kazak patientswith hypertension complicated with left ventricular hypertrophy using high-density DNA methylation chips. Methods: Three Uygur and three Kazak patients with hypertension complicated with left ventricular hypertrophy were selected for DNA extraction and bisulfite conversion. Left ventricular hypertrophy was defined by echo Penn convention as left ventricular mass index $\geq 115 \mathrm{~g} / \mathrm{m} 2$ in men and $\geq 95 \mathrm{~g} / \mathrm{m} 2$ in women. Illumina HD450K Infmium Methylation BeadChip was applied to detect 450,000 methylation sites of the whole human genome.The cluster analysis and significance test were carried out to analyze the different methylation levelsin Uygur and Kazak patients with left ventricular hypertrophy. The Delta Data were screened for statistics. Results: A total of 236genes (including 84 hypermethylated and 152 hypomethylated) showed significant differences in the degree of methylation between Uygur and Kazak patients. The average methylation level of significantly different genes was higher in the experimental group compared with the control group.The chromosome distribution of aberrant methylationgenes was quite even. The cluster analysis of the candidate genes showed thataberrant methylation genes in Uygur and Kazak patients with hypertension complicated with left ventricular hypertrophy mainly participated in cell adhesion,signal transduction, and cell cycle.Conclusions:Different methylation genes were found in Uygur and Kazak patients with hypertension complicated with myocardial hypertrophy, providing the basis for further studies on the molecular mechanism and prevention and treatment ofhypertension complicated with target organ damage among different nationalities in Xinjiang.

\section{Background}

Kazak nationals in Xinjiang is a high-risk group of hypertension. The prevalence of hypertension and blood pressure among adolescents is higheramong Kazak nationals than among Uygur nationals, and the prevalence increases more obviously after the age of 25 years ${ }^{[1]}$. Data show that the increase in the relative risk of hypertension and the degree of blood pressure is higher in Kazak people than in Uygur people ${ }^{[2,3]}$, but the prevalence of myocardial hypertrophy, heart failure, and atrial fibrillation caused by hypertension is lower than thatin Uygur people.

The cases of 12,449 patients with hypertension at the First Affiliated Hospital of Xinjiang Medical University in May 2015 were reviewed. Themerged disease prevalence of Kazak revealed ethnicity-related differences in patients with left ventricular hypertrophy ${ }^{[4]}$. The blood pressure level could not fully explain the differences in hypertension complicated by myocardial hypertrophy ${ }^{[5]}$.

The role played by DNA methylation in the occurrence and development of hypertension and target organ damage in different ethnic groups and its pathophysiological mechanism need further exploration and research.

\section{Methods}




\subsection{Participants}

Uygur and Kazak patients with hypertension complicated with left ventricular hypertrophy admitted to the First Affiliated Hospital of Xinjiang Medical University in 2016 were selected for diagnosing primary hypertension according to the diagnostic criteria of hypertension in the guidelines for the prevention and treatment of hypertension in China $2018^{[6]}$. Left ventricular hypertrophy was defined as left ventricular mass index $110 \mathrm{~g} / \mathrm{m}^{2}$ in males and $95 \mathrm{~g} / \mathrm{m}^{2}$ in females measured by color Doppler. All patients diagnosed with secondary hypertension based on medical history inquiry, physical examination, and related auxiliary examination and those not associated with severe coronary heart disease, cardiac valvular disease, cardiomyopathy, pulmonary heart disease, hyperthyroidism, diabetes, hepatic and renal insufficiency, malignant tumors, and connective tissue disease were excluded from the study. Three Uygur and three Kazak patients with matching age, gender, hypertension course, blood pressure level, and left ventricular quality index were selected, with a male-to-female ratio of 2:1. The average age of Uygur and Kazak patients was 52.7 and 52.1 years, respectively.

\subsection{Study methods}

\subsubsection{Specimen collection: The venous blood of patients who fasted for more than 12 hours was} collected. Blood cells and plasma were rapidly separated after specimen collection and stored at-70 ${ }^{\circ} \mathrm{C}$. All blood samples were obtained with the informed consent of the participants and approved by the hospital ethics committee.

\subsubsection{Echocardiography: Echocardiography was performed using Philips iE33 color Doppler ultrasound} diagnostic instrument, with probe frequency ranging from 5 to $10 \mathrm{MHz}$.The end-diastolic ventricular septal thickness, interventricular septum thickness (IVST), left ventricular posterior wall thickness(LVPWT), left ventricular end-diastolic diameter (LVEDD), left atrial dimension, and ejection fraction were evaluated. The Devereux formula was used to calculate the left ventricular mass (LVM): $0.8 \times 1.04 \times[($ LVEDD+ IVST+ LVPWT)3-LVEDD3]+0.6g; body surface area (BSA):BSA(male) $=0.0057$ height $(\mathrm{cm})+0.0121$ weight $(\mathrm{kg})+0.0882$; and BSA (female) $=0.0073$ height $(\mathrm{cm})+0.0127$ weight $(\mathrm{kg})+0.2106$. Left ventricular mass index (LVMI) was calculated as follows: LVMI = LVM/BSA.

\subsubsection{Genomic DNA extraction and DNA methylation level detection: The whole genomic DNA of} peripheral blood was extracted using the Tiangen Kit (TiangenBiochemical Technology (Beijing) Co. Ltd.). Sulfite transformation, denaturation, and amplification of genomic DNA were carried out by the optimization method using Zymo EZ DNA Methylation Kit officially recommended by Illumina, and then the products were digested and amplified using random endonuclease. The DNA fragments were hybridized with Infinium Methylation BeadChip, and the specific capture probe on the chip was combined with complementary enzyme digestion gene fragments. Following overnight hybridization, single base extension and staining were carried out after cleaning and scanning.

1.2.4 Differential gene screening: The scanned raw data were used to generate the methylation level of each site of every sample using Genome Studio software. After deviation correction and site filtering, the 
methylation level results were obtained. For paired methylation analysis of the two groups of samples, the Diff score $>13$ or $<-13$ and Delta_Beta $>0.2$ or $<-0.2$ were used as the threshold to screen the different methylation sites. The Gene Ontology (GO) and Kyoto Encyclopedia of Genes and Genomes (KEGG) signaling pathways were analyzed using the DAVID database for significantly different methylated genes.

\section{Results}

\subsection{Screening of differential methylation sites and genes}

The beta score was determined using the $\log ($ Beta $) /(1$ - Beta) formula.Benjaminiand Hochberg method was used for calculating FDR. The $P$ value was less than 0.05 , and the average beta score difference between the two groups was greater than 0.2. The differentially methylated regions (DMRs) were screened.The results showed that DMRs had 236 genes (84 with increased methylation degree and 152 with decreased methylation degree (Figures. 1 and 2).

Figure 3 shows the volcanic map of the degree of difference between the two sample groups. Red is the different methylation sites. The figure reveals the amount and distribution of DMRs.

Unsupervised hierarchical clustering was performed for different methylation sites. The distance between the two samples was calculated to form the distance matrix. The two closest to each other were merged into a new class. The distance between the new class and the current class was calculated, and then the distance between the new class and the current class was merged and calculated until only one class remained. The direct correlation of the sample was calculated based on the expression of the selected differential methylation sites. In Figure 4,W1, W2, and W3 denote hypertension with myocardial hypertrophy in Uygur patients while $\mathrm{H} 1, \mathrm{H} 2$, and $\mathrm{H} 3$ denote hypertension with myocardial hypertrophy in Kazak patients. The red is the hypermethylation site, and the green is the hypomethylation site. The results showed that most loci in the Kazak group showed hypermethylation (red) while most loci in the Uygur group showed decreased methylation (green).

\subsection{Functional analysis of differential methylation sites}

\subsubsection{GO function enrichment analysis}

The $\mathrm{GO}$ analysis was performed on the genes corresponding to different methylation sites, and the functions of the genes were described. GO included three major parts: biological process, cellular component, and molecular function, leading to three kinds of results. The number of differentially expressed genes in each entry was counted, and the functional categories of 3639 differentially expressed methylated genes were counted (FDR< 0.05).Differences between the methylation of gene function mainly focused on ion channels (Figure. 5). The ethnicity-related differences in hypertension with left ventricular hypertrophy might be related to the low methylation status promoting ventricular hypertrophy (Table 1) 
The database was used to analyze the genes corresponding to different methylation loci, and the significance of gene enrichment in each item was calculated using statistical tests. The analysis of the differentially expressed genes revealed entries enriched with differentially expressed genes. The differentially expressed genes in different samples that might be related to the changes in cell pathways were searched. It was found that these gene pathways mainly focused on endocytosis, cell adhesion, immune system, carbohydrate metabolism, and other aspects (Figure. 6)

\section{Discussion}

The etiology of primary hypertension is closely related to genetic and environmental factors. Of these, genetic factors, namely susceptibility genes, may play a decisive role in the occurrence of hypertension ${ }^{[7,8]}$. Relevant studies suggested ethnic and national differences in left ventricular remodeling in patients with essential hypertension. The left ventricular remodeling was higher in Uygur patients than in the Han and Kazak patients. Also, the tolerance to high blood pressure load of Kazak patients was high, but left ventricular hypertrophy was lower than that inUygur patients ${ }^{[9]}$. With the development of molecular genetics and molecular biology technology in recent years, the etiology of hypertension has been analyzed from the perspective of genes. The incidence of hypertension may be related to some susceptible genes ${ }^{[10]}$. The pathogenesis and pathological process of hypertension are more complex and difficult to explain. Consequently, the research on DNA methylation modification and hypertension has not achieved much progress. However, some studiessuggested that abnormal DNA methylation modification might affect the expression of some candidate genes related to hypertension and ultimately play a role in the disease process ${ }^{[11,12]}$.

\section{Conclusions}

In this study, the genome-wide methylation levels were compared among Uygur and Kazak patients with hypertension complicated with left ventricular hypertrophy, revealingwhole-genome differences between methylation sites of the two groups. The 443 different methylation sites were distributed in 236 genes (beta score $>0.2, P<0.05$ ). Further, 84 genes had increased methylation level, and 152 had decreased methylation level.The GO and KEGG function analyses of hypermethylated genes showed that the gene functions with methylation differences were mainly concentrated in the cell signaling pathway, stimulation response regulation, immune system process, cell adhesion molecules, and cell cycle apoptosis. Comparing the microarray results of Uygur and Kazak patients with myocardial hypertrophy showed that a variety of genes with elevated methylation level, such as IGFALS and ATP8A2, inhibited the occurrence and development of myocardial hypertrophy. In addition, a variety of genes with reduced methylation level, such as ACTN2 in the myocardium and skeletal muscle and IGF1R related to lipid metabolism and protein decomposition, promoted the occurrence of hypertensive left ventricular hypertrophy. The level of methylation negatively correlated with the expression of these genes, and the change in methylation level of each gene led to the enhancement of the biological function of promoting 
myocardial hypertrophy or the decrease in the biological function of inhibiting myocardial hypertrophy, thus promoting the development of hypertensive myocardial hypertrophy.

This study was conducted to compare the methylation level in hypertension with myocardial hypertrophy among different ethnic groups (Uygur and Kazak). However, due to the small sample size, the differential methylation loci or genes screened in this study could not represent the common characteristics of this ethnic group. Therefore, large-sample studies should compare the specific sites of DNA methylation in the Uygur and Kazak patients with hypertension to highlight the differences in patients with myocardial hypertrophy, thusproviding new testsfor the early diagnosis of myocardial hypertrophy.

\section{Abbreviations}

IVST-Interventricular Septum Thickness

LVPWT-Left Ventricular Posterior Wall Thickness

LVEDD:-Left Ventricular End-diastolic Diameter

LVM-Left Ventricular Mass

BSA:-Body Surface Area

LVMI:-Left Ventricular Mass Index

DMRs- Differentially Methylated Regions

\section{Declarations}

Ethics approval and consent to participate: This study was approved by the ethics committee of the first affiliated hospital of xinjiang medical university. And the consent I received from the study participants was in writing. This study abides by $<$ The international Helsinki declaration $>$, <Measures for ethical review of biomedical research involving human beings (trial)> and GCP and other relevant ethical principles formulated by Chinese health authorities. Ethical approval number:20140905-08.

Consent for Publication: Not applicable

Availability of data and material: The reported [figure and table] data were used to support this study and are available at [The original data]. These prior studies (and data support) are cited at relevant places within the text as references.

Competing interests: The authors declare that they have no competing interests. 
Funding: $\quad$ Natural science foundation of xinjiang autonomous region. Financial support was provided for the design of research, the collection, analysis and interpretation of data.

Authors' contributions:Xi Chen and Xinjuan Xu have made substantial contributions to the conception and design of the work, and $\mathrm{Na}$ Zhang has made contributions to the acquisition, analysis and interpretation of data, so Xi Chen and Na zhang are the joint first author, Xiaohui Liang and Guangmei Hu have revised the draft of the article.

Acknowledgements: Not Applicable.

\section{References}

[1]FenLiu. Cardiac Complications of Hypertension [J]. Chinese journal of practical internal medicine,2002,22(4):198-199.

[2] National blood pressure sampling cooperative group. Incidence and trend of hypertension in Chinese population [J]. Hypertension,1995,3(suppl 1):7-13.

[3] Fen Liu, YitongMa,Yining Yang, et al. Epidemiological investigation of hypertension in different ethnic groups in xinjiang [J]. Chinese journal of medicine, 2010, (46) : 3259-3263.

[4] Liyun Lin, XinjuanXu,Xi Chen, et al. Left ventricular remodeling in patients with primary hypertension in xinjiang Han, Uygur and Kazak ethnic groups and the differences in related influencing factors [J].Chinese journal of hypertension, 2016,24 (6) :564-571.

[5]Heineke J, Molkentin JD. Regulation of cardiac hypertrophy by intracellular signalling pathways[J]. Nature reviews: molecular cell biology,2006,8(8).

[6]Chinese committee for the revision of guidelines for the prevention and treatment of hypertension, Hypertension alliance (China), Chinese society of cardiology,et al.Guidelines for hypertension prevention and treatment in China (2018) [J]. Chinese Journal of Cardiovascular Medicine. 2019,(01):1007-5410.

[7] Friso S, Carvajal CA, Fardella CE, et al. Epigenetics and arterial hypertension:the challenge of emerging evidence[J].Transl Res,2015,165(1):154-165.

[8] Goh L, Murphy SK, MuhkerjeeS,et al. Genomic sweeping for hypermethylated genes[J]. Bioinformatics,2007,3(3):281-288.

[9] Ohta M®Kasamaki Y®Ozawa Y,etal.Combined effects of blood pressure and aldosterone on cardiac left ventricular mass index-ethnic difference between Kazakh囚Uygur and Han bsubjects[J]. World J Cardiovasc,2014,4:99-105.

[10]XiaorongWang,Ming Ren.Research progress of three gene polymorphisms and essential hypertension[J]. Chinese Journal of Geriatric Heart Brain and Vessel Diseases, 2019,21(1):1009-0126. 
[11] Lim SS,VosT,FlaxmanAD,etal.Acomparatinv risk assessment of burden of disease and injury altributable to 67 risk factous and risk factor clusters in 21 regions,1990-2010:a systematic analysis for the Gloval Burden of Disease Study 2010[J].Lancet,2012,380(9859):2224-2260.

[12]Suzuki MM, Bird A. DNA methylation landscapes: provocative insights from epigenomics.[J]. Nature reviews. Genetics,2008,6(6):465-476.

\section{Tables}

Table 1. Differentially methylated genes associated with left ventricular hypertrophy in Uygur and Kazak patients with hypertension

\begin{tabular}{lccc}
\hline Gene Chip results $\quad$ Effect on left ventricular hypertrophy Beta difference & $P$ \\
\hline IGFALS High methylation Inhibits left ventricular hypertrophy & 0.379 & $4.20 \times 10^{-8}$ \\
\hline ACTN2 Low methylation Promotes left ventricular hypertrophy & -0.601 & 0.023 \\
\hline IGF 1R Low methylation Promotes left ventricular hypertrophy & -0.293 & 0.018 \\
\hline ATP8A2 High methylation Inhibits left ventricular hypertrophy & 0.357 & $2.85 \times 10^{-7}$ \\
\hline NCOR2 High methylation Inhibits left ventricular hypertrophy & 0.637 & $2.48 \times 10^{-8}$ \\
\hline SMG6 Low methylation Promotes left ventricular hypertrophy & -0.293 & $2.25 \times 10^{-5}$ \\
\hline
\end{tabular}

\section{Figures}




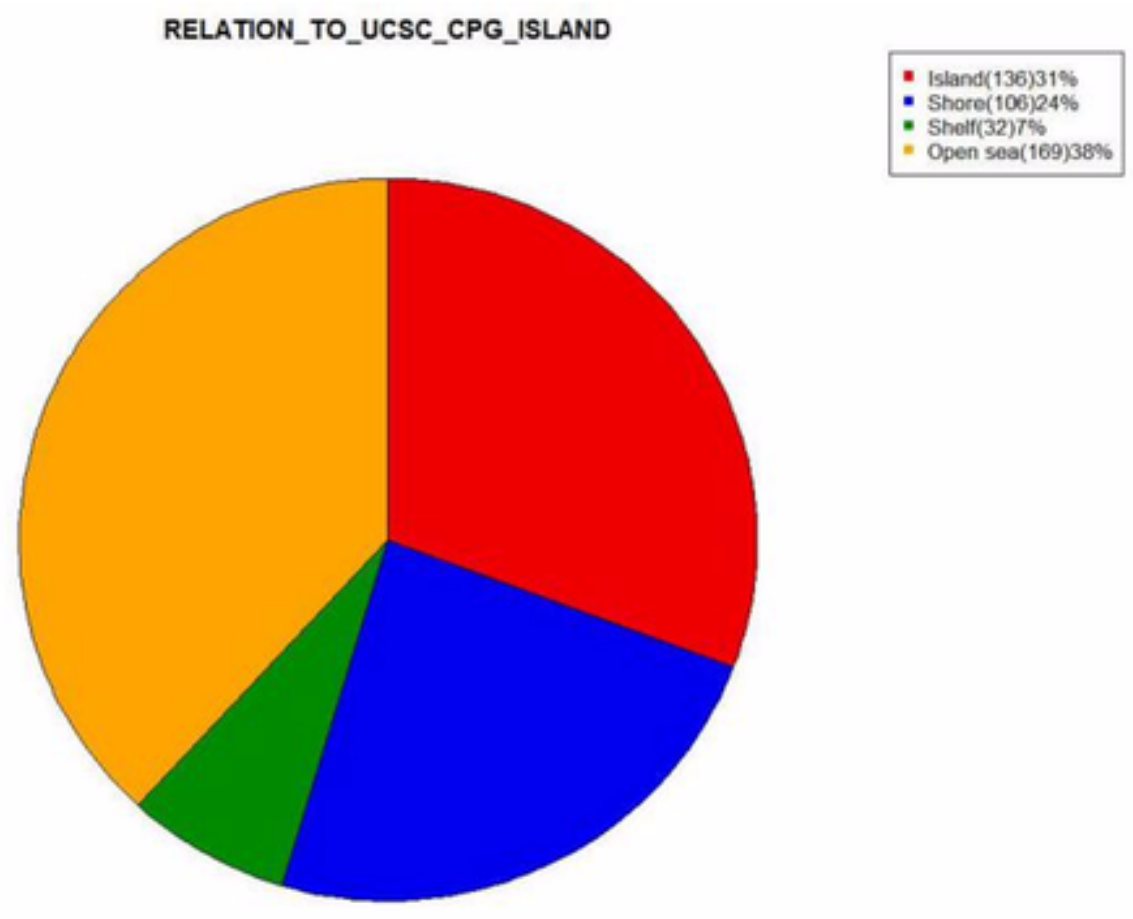

Figure 1

Different methylation sites of Uygur and Kazak groups.

152
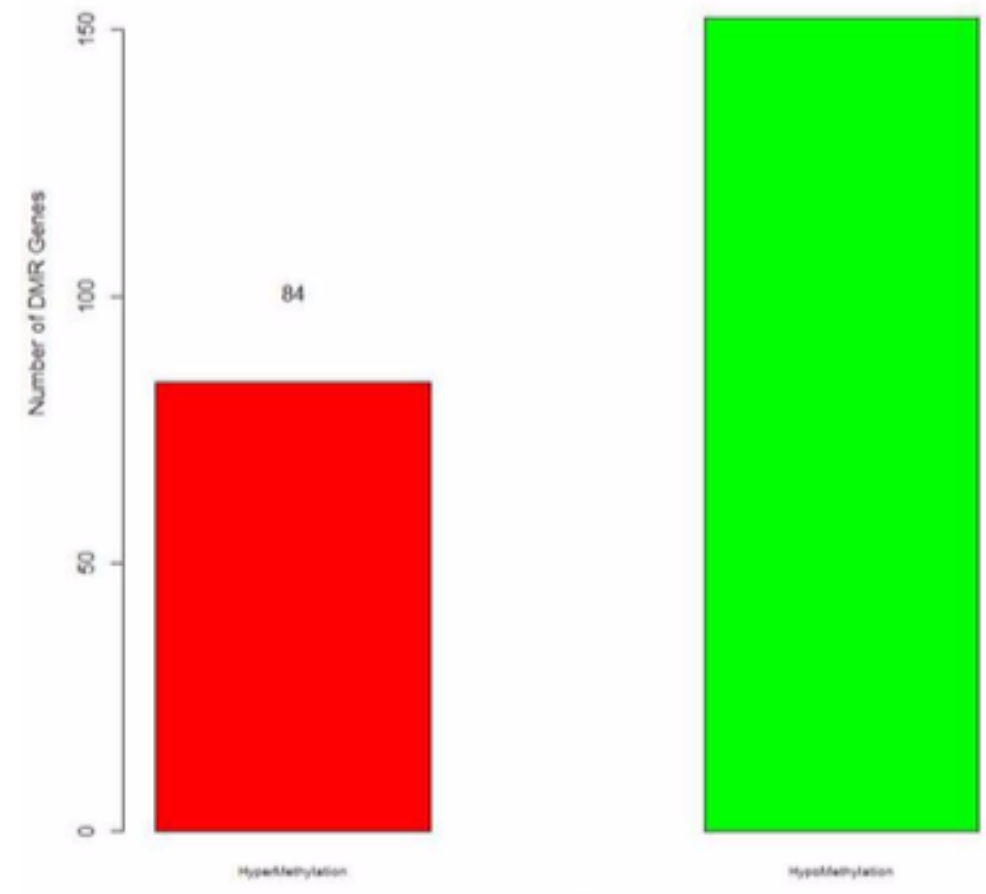

Figure 2 
Different methylation genes of Uygur and Kazak groups.

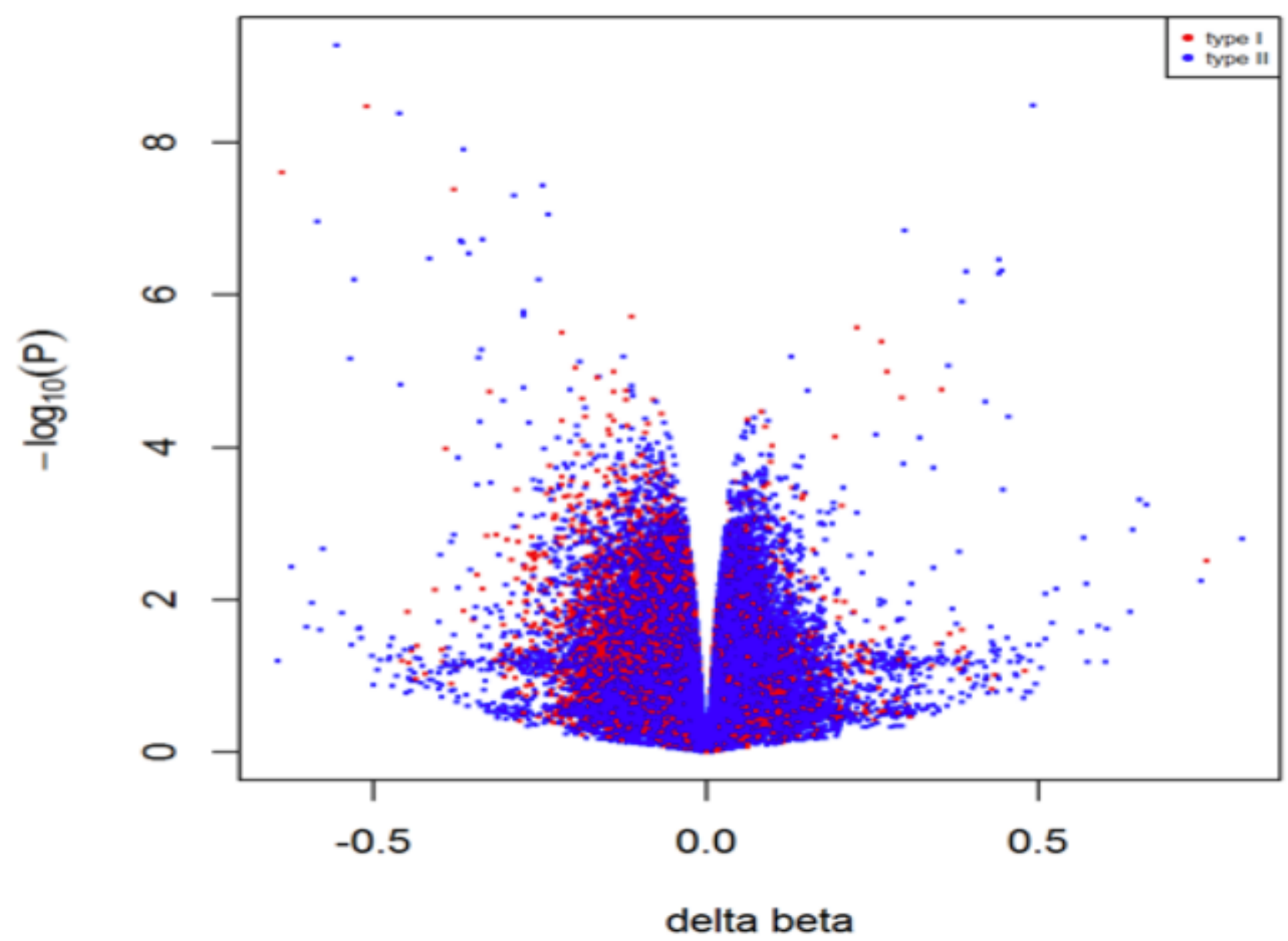

Figure 3

Volcanic map of the degree of difference between the two sample groups. 


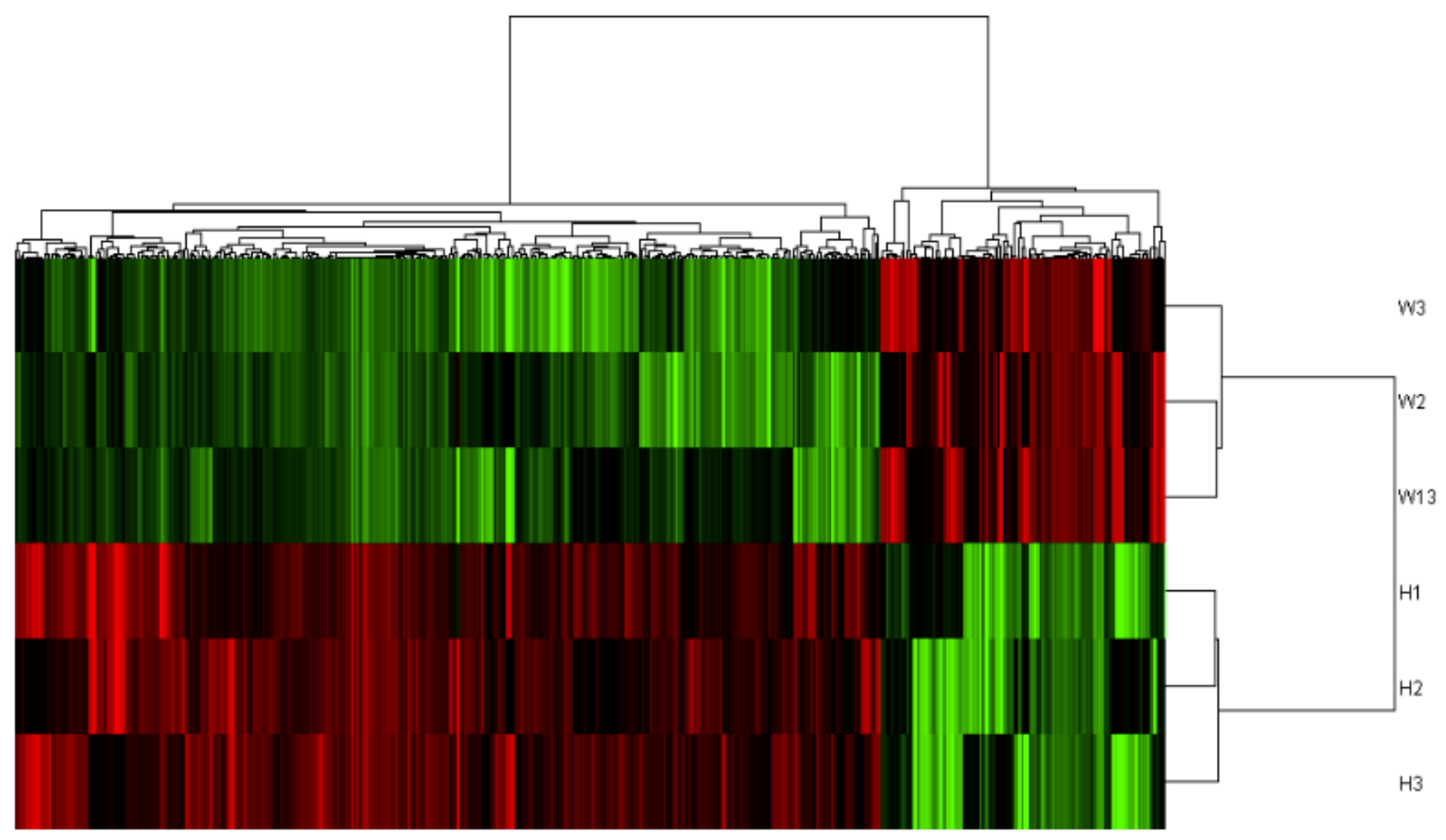

\section{Figure 4}

Cluster diagram of different methylation sites and samples.

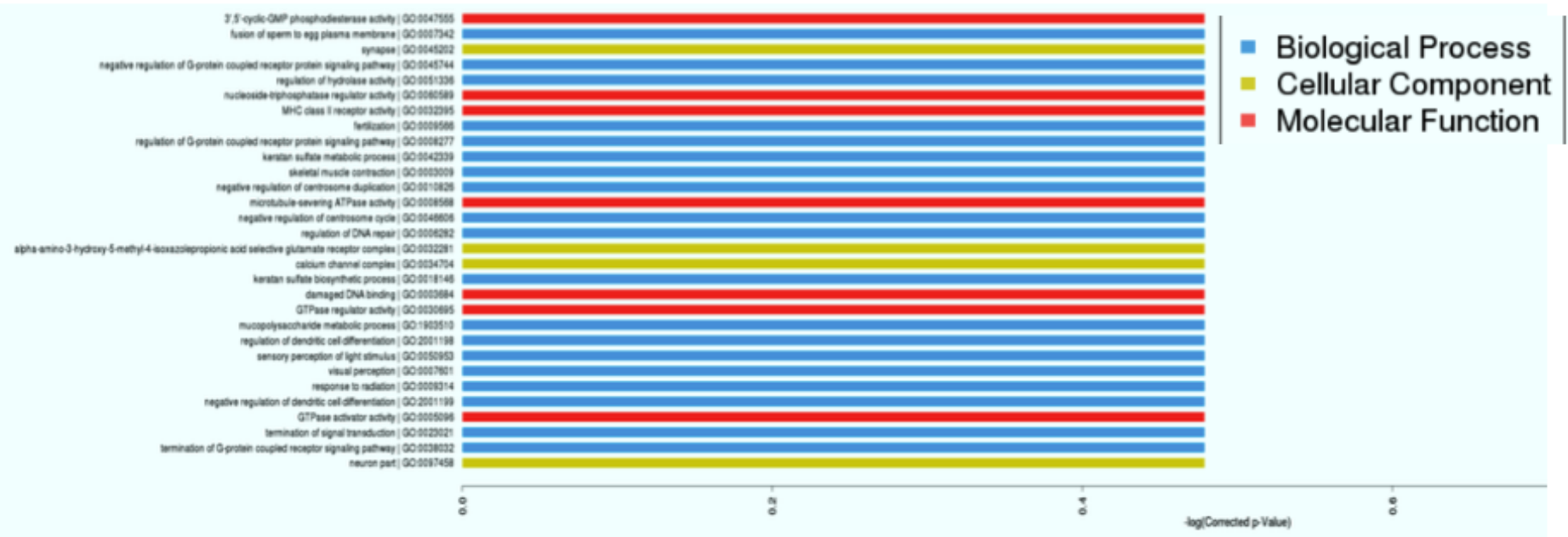

Figure 5

GO function enrichment diagram. 


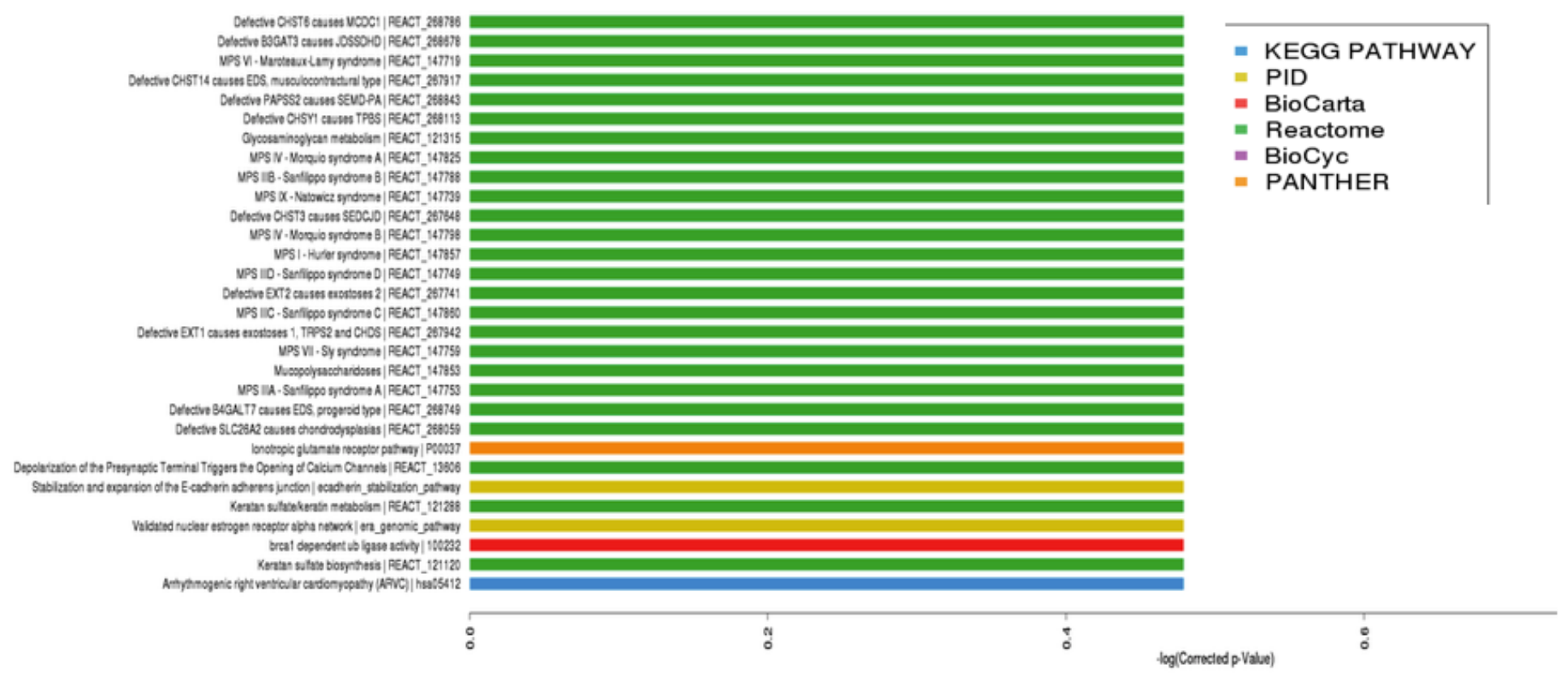

Figure 6

Pathway enrichment map.

\section{Supplementary Files}

This is a list of supplementary files associated with this preprint. Click to download.

- renamed02528.idat

- renamed0e3c1.idat

- renamedOfacd.idat

- renameddc286.idat

- renamed150cc.sdf 